Olivier Giraud, Nikola Tietze in Zusammenarbeit mit Camille Noûs

Version provisoire du texte final, paru dans Europäisches Zentrum für Föderalismus-Forschung Tübingen (EZFF) (éd.): Jahrbuch des Föderalismus 2021. Föderalismus, Subsidiarität und Regionen in Europa. Baden-Baden: Nomos 2021, pp. 85-98

\title{
Die zentralistische Organisation des öffentlichen Handelns in Frankreich konfrontiert mit den Herausforderungen der Covid-19-Pandemie
}

Mit mehr als 1.300.000 Todesfällen im Zusammenhang mit dem Coronavirus SARS-CoV-2 liegt Frankreich gegenwärtig zwischen den europäischen Ländern, in denen die Zahl der Todesfälle 1.600 pro eine Million Einwohner überschreitet (z. B. Italien und Großbritannien), und denen, wo (etwa Deutschland) die Anzahl der Todesfälle noch unter 1.000.000 liegt. Insofern gehört es in Europa zu den Ländern, die relativ stark von der Covid-19-Pandemie betroffen sind. Dieser traurige Befund wirft die Frage auf, ob das staatliche und institutionelle Regime, insbesondere das des Gesundheitssystems, den Verlauf der Pandemie in Frankreich beeinflusst hat. Im Folgenden versuchen wir, Elemente für eine Antwort auf diese Frage herauszuarbeiten. Wir nehmen hierfür das öffentliche Handeln in den Blick, durch das staatlich definierte public policies, Behörden und gemeinnützige oder gewinnorientierte Interessengruppen gesellschaftliche Solidarität produzieren. In einer steuerungstheoretischen Perspektive untersuchen wir die entsprechenden Ebenen des öffentlichen Handelns (policy scales). Hierbei berücksichtigen wir insbesondere den Gesundheits- und Sozialbereich und stellen unsere Untersuchung in einen Bezug zum Pandemieverlauf ab Winter 2019/2020 bis Juli 2021.

Unter den Ebenen des öffentlichen Handelns verstehen wir Interaktionsräume, in denen öffentliche Dienstleistungen und Güter hergestellt werden. Die Ebenen des öffentlichen Handelns spiegeln sowohl die vertikale territoriale Ordnung zwischen den zentralstaatlichen Instanzen und dezentralen öffentlichen Einrichtungen in den Gebietskörperschaften als auch die horizontale Organisation der Beziehungen zwischen verschiedenen öffentlichen und privaten Akteuren wider. ${ }^{1}$ Als Interaktionsräume, die durch vielfältige Interdependenzen institutioneller, hierarchischer, finanzieller oder professioneller Art miteinander verflochten sind, entstehen zwischen den vertikalen und horizontalen Ebenen Maßstabsverschiebungen,

\footnotetext{
${ }^{1}$ Vgl. Kazepov, Yuri 2011: Rescaling in der Sozialpolitik: Die neue Rolle lokaler Wohlfahrtssysteme in europäischen Staaten, in: Hanesch, Walter (Hrsg.): Die Zukunft der "Sozialen Stadt". Strategien gegen soziale Spaltung und Armut in den Kommunen, Wiesbaden, S. 115-153, für unser Verständnis der Ebenen des öffentlichen Handelns insbesondere S. 119-122.
} 
welche wir mit Jacques Revel als jeux d'échelles bezeichnen. ${ }^{2}$ Durch die jeux d'échelles im öffentlichen Handeln werden Prozesse angestoßen, die die Fähigkeit der Akteure zu handeln und insofern deren Steuerungskapazitäten beeinflussen. Letztere nehmen wir im Anschluss an die Steuerungstheorie - und zwar sowohl in ihrer deutschen als auch französischen Version im Folgenden in den Blick. ${ }^{3}$

Die Zusammenhänge zwischen der institutionellen Architektur und der Fähigkeit der einzelnen Akteure, die medizinische Versorgung oder Impfung während der Covid-19-Krise zu organisieren, gesundheitlichen Schutz zu gewährleisten, Restriktionen zu definieren oder zu kontrollieren sind weder direkt noch eindeutig. ${ }^{4}$ Die Schwierigkeiten in der Entscheidungsfindung und Organisation der französischen Pandemiebekämpfung wie auch die Spannungen bei der Umsetzung der Anit-Covid-Maßnahmen verweisen gleichwohl auf solche Zusammenhänge, die bisher unter unterschiedlichen Gesichtspunkten beschrieben worden sind, z. B. als mangelnde Vorbereitung des französischen Gesundheitssystems auf eine Pandemie, ${ }^{5}$ als verspätetes Einführen von Eindämmungsmaßnahmen, ${ }^{6}$ als Fragmentierung des gesundheitspolitischen Handelns ${ }^{7}$ wie auch als plethorische Vervielfältigung der Instanzen im Krisenmanagement ${ }^{8}$. Wir stützen uns auf diese verschiedenen Erklärungsansätze und heben in dem folgenden Beitrag spezifische Mechanismen in der französischen Pandemiebekämpfung hervor. Um ein Licht auf die Zusammenhänge zwischen den institutionellen Strukturen des französischen Staatsregimes und dem Verlauf der Covid-19-Krise zu werfen, ziehen wir im analytischen Blick auf die Ebenen des öffentlichen Handelns und den hiermit verbundenen jeux d'échelles in einer

\footnotetext{
${ }^{2}$ Zu den jeux d'échelles vgl. Revel, Jacques 1996: Micro-analyse et construction du social, in Revel, Jacques (Hrsg.): Jeux d'échelles. La micro-analyse à l'expérience, Paris, S. 15-36; Tietze, Nikola 2019: Jeux d'échelles. Reflexionen über ein methodisches Prinzip und eine analytische Beschreibungskategorie, in: Glossar der Missverständnisse, Berlin. https://rm2.hypotheses.org/1046 (24.07.20021).

${ }^{3}$ Mayntz, Renate/Scharpf, Fritz W. 1995: Gesellschaftliche Selbstregelung und politische Steuerung, Frankfurt am Main; Reynaud, Jean-Daniel 1989: Les règles du jeu - L'action collective et la régulation sociale, Paris. Friedberg, Erhard 1993: Le pouvoir et la règle, Paris.

${ }^{4}$ Bandelow Nils C./Hassenteufel, Patrick/Hornung, Johanna 2021: Patterns of Democracy Matter in the COVID19 Crisis. A comparison of French and German Policy Processes, in: International Review of Public Policy Jg. 3, Nr. 1, S. 1-18.

${ }^{5}$ Hassenteufel, Patrick 2020: Handling the COVID-19 crisis in France: Paradoxes of a centralized state-led health system, in: European Policy Analysis Nr. 6, S. 170-179.

${ }^{6}$ Rosental, Paul-André 2020: Les trente premiers jours. Leçons comparatives de la lutte contre la pandémie de coronavirus, in: Lazar, Marc/Plantin, Guillaume/Ragot, Xavier (Hrsg.): Le monde d'aujourd'hui. Les sciences sociales au temps de la Covid, Paris, S. 23-44.

${ }^{7}$ Giraud, Olivier/Tietze, Nikola/Toffanin, Tania/Noûs, Camille 2021: The scalar arrangements of three European public health systems facing the COVID-19 pandemic: Comparing France, Germany, and Italy, in: Culture, Practice \& Europeanization Jg.6, Nr. 1, S. 89-111.

${ }^{8}$ Bergeron, Henri/Borraz, Olivier/Castel, Patrick/Dedieu, François 2020: COVID-19. Une crise organisationnelle, Paris.
} 
steuerungstheoretischen Perspektive folgende Punkte genauer in Betracht: Nach einer kurzen Einordnung des französischen Zentralismus und einer skizzenhaften Einführung in die Chronologie des französischen Krisenmanagements betrachten wir in einem ersten Teil die territoriale Organisation der staatlichen Ordnung im Allgemeinen und des öffentlichen Gesundheitsbereichs im Besonderen (1.). Im zweiten Teil richten wir den Blick auf die Strukturen der wissenschaftlichen Beratung und Entscheidungsfindung während des französischen Krisenmanagements (2.). Es geht uns hier um die Modalitäten, in denen die Maßnahmen für Gesundheitsschutz - Prävention, Pflege und Forschung - und zivile Sicherheit umgesetzt worden sind. Schließlich wenden wir uns im dritten Teil den Instrumenten des öffentlichen Handelns und der kritischen Reaktionen auf das Krisenmanagement zu (3.).

\section{Die territoriale Ordnung des öffentlichen Handelns in Frankreich: ein komplexes und gleichzeitig unsicheres Verhältnis zwischen Zentrum und Peripherie}

In seinem Vergleich westlicher Staatssysteme bezeichnet der Politikwissenschaftler und Föderalismusspezialist, Daniel Elazar, Frankreich als das Beispiel für ein hierarchisches Organisationsmodell. ${ }^{9}$ Er macht deutlich, dass die in der Abfolge der französischen politischen Regime, insbesondere der Republiken, die Machtverhältnisse nicht wirklich verändert und vor allem das pyramidale und hierarchische Muster beibehalten worden ist. ${ }^{10}$ Dieses Muster, das auf einer starren Kette von Anweisungen basiert, steht in einem deutlichen Gegensatz zum Föderalismus. Auch das aktuelle Regime der fünften französischen Republik ist - wenn nicht gar in verstärktem Maße - ein stark zentralisiertes staatliches und demokratisches System, in dem der Präsident der Republik und die von ihm ernannte Regierung Dreh- und Angelpunkt der Wahlen, der Entscheidungsfindung und Steuerung darstellen.

Die Covid-19-Krise hat wie durch ein Vergrößerungsglas die zentrale Position der französischen doppelköpfigen Exekutive, Präsident-Regierung, sichtbar gemacht. Die neun Ansprachen von Emmanuel Macron im Rahmen der Bewältigung der Covid-19-Krise, acht Adresses aux Français plus die traditionellen Neujahrsgrüße vom 31. Dezember 2020, jeweils gefolgt von Regierungserklärungen durch die beiden in dem Zeitraum amtierenden Premierminister (zunächst Edouard Philippe, dann Jean Castex), die die Einschränkungen

\footnotetext{
${ }^{9}$ Elazar, Daniel J. 1997: Contrasting Unitary and Federal Systems, in: International Political Science Review / Revue internationale de science politique Jg.18, Nr. 3: 237-251.:

${ }^{10}$ Ebenda, S. 241.
} 
oder Lockerungen spezifizierten, haben in Frankreich den Rhythmus im Verlauf der Covid19-Pandemie bestimmt und jener eine spezifisch französische Chronologie aufgedrückt. Diese Chronologie, die den Verlautbarungen der Weltgesundheitsorganisation (WHO) über die Verbreitung von Sars-Cov-2 deutlich hinterherhinkte und mehr oder weniger mit der Agenda der Maßnahmen in Italien, Deutschland und anderen Mitgliedsstaaten der Europäischen Union (EU) übereinstimmte, hat in Frankreich nicht nur die Erfahrungen der Wohnbevölkerung mit der Pandemie geprägt, sondern auch die Interaktionsräume, die die Ebenen des öffentlichen Handelns darstellen, beeinflusst. Sie ist durch drei Momente der Steuerung strukturiert und in drei Phasen gegliedert:

Das erste Moment, dem eine Phase der Verharmlosung durch die Exekutive voranging, setzten die ersten beiden Ansprachen Emmanuel Macrons - am 12. März 2020, in der er die Bedeutung des Sozialstaats, der Wissenschaft und der Solidarität betonte, und am 16. März 2020, in der er einen Tag nach dem ersten Wahlgang der Kommunalwahlen in einem kriegerischen Ton die Bürger zum Respekt des Lockdowns (confinement) und seines Regelwerk aufrief. Die erste Phase endete mit der Aufhebung des Lockdowns (Ende des déconfinement), am 14. Juni 2020, was den Weg zum zweiten Wahlgang der Kommunalwahlen, am 28. Juni 2020 (ursprünglich geplant für den 29. März 2020 geplant), frei gab.

Die zweite Phase begann am 14. Oktober 2020 mit der Einführung des Steuerungsmoments einer nächtlichen Ausgangssperre (couvre-feu) in der Region Ile-de-France und acht urbanen Metropolen, zunächst von 21 Uhr bis 6 Uhr, die dann auf das gesamte Staatsgebiet und auf 19 Uhr und schließlich auf $18 \mathrm{Uhr}$ bis $6 \mathrm{Uhr}$ ausgeweitet wurde. In die zweite Phase fiel ein zweiter nationaler Lockdown (30. Oktober 2020 bis 15. Dezember 2020), der weniger strikt als der erste war, u. a. weil Schulen und die meisten Behörden des sozialen Sektors geöffnet blieben.

Das dritte Moment zentralstaatlicher Steuerung ist gekennzeichnet durch eine Kombination aus der Aufrechterhaltung oder Verstärkung bestimmter Restriktionen, der Ankurbelung des Wirtschaftslebens und der Organisation der Impfkampagne. Begleitet von einer öffentlichen Auseinandersetzung über die wirtschaftlichen, sozialen und psychologischen Auswirkungen der Covid-19-Eindämmerungsmaßnahmen, führte diese Kombination in einen dritten Lockdown (vom 3. April 2021 bis zum 3. Mai 2021) und setzt sich bis heute im Konflikt über den Gesundheitspass (pass sanitaire) und die Impfpflicht fort.

Seit den frühen 1980er Jahren haben die sozialistischen wie konservativen Regierungen Frankreichs immer wieder Territorialreformen beschlossen, die das zentralistische Staats- und 
Demokratiesystem spätestens seit den 2000er Jahren zu einer komplexen, sich ständig verändernden und für die Bürger unleserlichen institutionellen und organisatorischen Architektur gemacht haben. ${ }^{11}$ In einer nunmehr fast 40 Jahre andauernden de- und rezentralisierenden Gliederung der Gebietskörperschaften bzw. Einführung neuer territorialer Verbandsformen (z. B. communauté d'agglomération, oder métropoles) sind unter dem politischen Schlagwort décentralisation Kompetenzen reorganisiert, Kommunikationskanäle restrukturiert und Verwaltungswege transformiert worden. Die Spannungen und Konflikte, die die Territorialreformen begleitet haben, ${ }^{12}$ verweisen gleichwohl auf eine Reihe von Dysfunktionalitäten. Letztere gehen auf mehrere Faktoren zurück.

Erstens sind einheitliche und zentralistische Normen in der Konzeption der französischen Staatsbürgerschaft fundamental und werden von den Bürgern als eine konkreten Form der Gleichheit verstanden. ${ }^{13}$ Insofern provozieren die Anerkennung territorialer oder anderer sozialer Besonderheiten wie auch die Differenzierung von Regeln oder Maßnahmen je nach territorialen oder gesellschaftlichen Bedingungen Ambivalenzen im Hinblick auf das französische Gleichheitsverständnis. Im Krisenmanagement der Covid-19-Krise sind solche Ambivalenzen als Steuerungsprobleme in den immer wieder aufbrechenden Spannungen zwischen Bürgermeistern und Präfekten deutlich zu Tage getreten. Während des ersten Lockdowns kam es vermehrt zu Konflikten im Zusammenhang mit der Versorgung mit Masken und Schutzausrüstung, die Bürgermeister für die Einwohner ihrer jeweiligen Stadt bestellt, jedoch von zentralstaatlichen Stellen vor der Auslieferung beschlagnahmt wurden. Nicht zuletzt stehen die gegenwärtigen Konflikte über die Ausweitung des Gesundheitspasses auf Freizeit- und Kultureinrichtungen im Kontext der Ambivalenzen, die das an einheitliche und zentralistische Normen gebundene Gleichheitsverständnis hervorrufen können. Sie zeigen zudem, dass dieses Gleichheitsverständnis - egal von welcher Konfliktpartei - jederzeit für die Politisierung von Steuerungsproblemen herangezogen werden kann.

Zweitens ist es für den Zentralstaat und die ihn bewohnenden hauts fonctionnaires (Beamte im höheren Dienst) schwierig, Kompetenzen vollständig auf eine Gebietskörperschaft zu übertragen. Letztendlich versuchen die zentralen Instanzen, die Mittel in der Hand zu behalten, die die Durchsetzung bestimmter nationalweit definierte Handlungsziele auch nach

\footnotetext{
${ }^{11}$ Vgl. insbesondere für die Präsidentschaften von Nicolas Sarkozy (2007-2012), François Hollande (2012-2017) und Emmanuel Macron (seit 2017): Halmes, Gregor 2020: Frankreich: territoriale Politik im Fadenkreuz der Krisenbewältigung?, in: Europäisches Zentrum für Föderalismus-Forschung Tübingen (EZFF) (Hrsg.): Jahrbuch des Föderalismus 2020. Föderalismus, Subsidiarität und Regionen in Europa, Baden-Baden, S. 291-306. 12 Ebenda.

${ }^{13}$ Vgl. Pollet, Gilles/Viard, Jean 2015: Quel avenir pour la maillage territorial français ?, in: Espaces et société, Nr. 160/161, S. 207-218.
} 
der Übertragung auf eine Gebietskörperschaft erlauben. Während des Managements der Covid-19-Krise wurde dies im Bereich der Pflege älterer und behinderter Menschen deutlich. Obwohl dieser Bereich in den Kompetenzbereich der Départements fällt, hat der Zentralstaat im Rahmen des Lockdowns und anhand der Definition strikter Besuchsregeln seine Kontrolle über diesen Bereich quasi zurückgewonnen.

Drittens hat die Tatsache, dass die Überwachung der öffentlichen Ausgaben bei zentralstaatlichen Instanzen liegt, dazu geführt, dass die Finanzaufsicht die Fähigkeit der Gebietskörperschaften, selbst Steuern zu erheben, eingeschränkt hat. Dadurch ist die Autonomie der Gebietskörperschaften begrenzt, über eigene Finanzen zu verfügen und auf der Basis einer lokalen oder regionalen Besteuerung eine eigene Infrastrukturpolitik zu betreiben.

Mit den Territorialreformen von $2014^{14}$ und $2015^{15}$ sollten die Kompetenzen der Gebietskörperschaften geklärt werden. Zum einen wurde den Départements und Regionen in einer Generalklausel Zuständigkeit gegeben. Dadurch können die Akteure dieser beiden Ebenen, in praktisch allen Bereichen des öffentlichen Handelns tätig zu werden. Diese allgemeine Kompetenz begünstigt jedoch die Redundanzen im öffentlichen Handeln und führt unter Umständen zu Widersprüchen zwischen verschiedenen Maßnahmen und Steuerungsversuchen auf den unterschiedlichen policy scales. Zum anderen haben die beiden Territorialreformen den Begriff des chef de file ausgeweitet. Wird eine Gebietskörperschaft zum chef de file in einem Bereich, besitzt sie den Vorrang in der Zuständigkeit für diesen Bereich. Die Region hat seitdem Entscheidungs- und Handlungskompetenz in den Bereichen Wirtschaftsentwicklung, Unternehmensförderung, Verkehr und Biodiversität, das Département wiederum die prioritäre Zuständigkeit in den Bereichen soziale Arbeit sowie Pflege, digitale Planung und territoriale Solidarität.

Die Gesundheitsüberwachung (sécurité sanitaire) liegt vornehmlich in der Verantwortung zentralstaatlicher Behörden, die in den Regionen durch die Präfekturen vertreten werden. Der Gesundheitsbereich ist ein Feld, in dem die Dezentralisierung des öffentlichen Handelns besonders zwiespältig und widersprüchlich ist. Zwar beziehen sich mehrere neuere Regelungen zur Gesundheitsreform auf die Dezentralisierung und die Gebietskörperschaften. Doch letztendlich haben sich die zentralisierten Strukturen der Gesundheitsverwaltung nicht wesentlich verändert. Niedergelassene Ärzte im Bereich des öffentlichen Gesundheitswesens

\footnotetext{
${ }^{14}$ La Loi du 27 janvier 2014 de modernisation de l'action publique territoriale et d'affirmation des métroples, genannt loi MAPTAM oder loi MAPAM.

${ }^{15}$ La Loi du 7 août 2015 portant nouvelle organisation territoriale de la République, genannt loi NOTRe.
} 
(médecine de ville oder auch Community Medicine) stehen vornehmlich in Kontakt mit den lokalen Vertretern der Caisse nationale d'assurance maladie, die eine besonders zentralisierte Einrichtung darstellt. Die Krankenhausmedizin hingegen liegt im Aufgabenbereich der regionalen Gesundheitsagenturen (Agences Régionales de Santé, ARS). Diese unterstehen jedoch in erster Linie dem Gesundheitsministerium in Paris. Die $A R S$ sind verantwortlich zum einen für die Koordination im Gesundheitsbereich mit den Behörden auf regionaler, departementaler und eventuell kommunaler Ebene und zum anderen für den Austausch mit den zentralstaatlichen Instanzen in den Gebietskörperschaften.

Während des Managements der Covid-19-Krise wurde deutlich, dass die Zusammenarbeit zwischen den ARS und den gebietskörperschaftlichen Behörden in Frankreich sehr ungleichmäßig entwickelt ist. Einen Austausch zwischen den ARS und den Präfekten in den Regionen bzw. anderen zentralstaatlichen Diensten auf den verschiedenen territorialen Ebenen hat es wiederum schlichtweg nicht gegeben. Hiervon zeugt vor allem die mangelhafte Koordination (insbesondere während des ersten Lockdowns) zwischen den Maßnahmen zur Gesundheitsüberwachung und denen des öffentlichen Gesundheitswesens im engeren Sinne.

\section{Strukturen der wissenschaftlichen Beratung und der Entscheidungsfindung im französischen Management der Covid-19-Krise}

Seit Jahren weisen Fachleute des öffentlichen Gesundheitswesens auf die strukturellen Schwächen dieses Bereichs in Frankreich hin, ${ }^{16}$ wo Krisen und Skandale sich seit langem aneinanderreihen - verseuchte Blutkonserven in den 1980er Jahren, gefährliche Medikamente in den 2000er Jahren, hohe Sterblichkeit durch die Hitzewelle im Sommer 2003 und jetzt die Krise der Covid-19-Pandemie. In der Folge dieser Krisen und Skandale haben sich die Reformversuche angehäuft und zur Etablierung von Kontrollstrukturen oder

Regulierungsorganen im Bereich der Gesundheit und Gesundheitsüberwachung geführt. ${ }^{17}$ Ein Beispiel hierfür ist Santé Publique France: eine staatliche Agentur, die direkt dem Gesundheitsministerium unterstellt und deren Aufgabe es ist, u. a. die Reaktion auf einen Gesundheitsnotstand zu organisieren. Die französischen Regierungen haben jedoch weitere Gremien geschaffen, wie den Haut Conseil de Santé Publique (Hohen Rat für öffentliche Gesundheit), der speziell dafür zuständig ist, Richtlinien für besondere gesundheitspolitische

\footnotetext{
${ }^{16} \mathrm{Vgl}$. Gilbert, Claude/Henry, Emmanuel 2009: Lire l'action publique au prisme de l'action publique des processus de définition des problèmes, in: Gilbert, Claude/Henry, Emmanuel (Hrsg.) Comment se construisent les problèmes de santé publique ?, Paris, S.7-33

${ }^{17}$ Bergeron/Borraz/Castel/Dedieu 2020 (Fn. 8).
} 
Herausforderungen zu formulieren. Zudem existieren auf einer eher operativen Ebene spezifische Verfahren zur Bekämpfung saisonaler Epidemien - insbesondere der Grippe. Bei Ausbruch der Covid-19-Pandemie im Winter 2019-2020 waren weder die für die Pandemiebekämpfung zuständigen Instanzen noch die Verantwortlichen für die Verfahren zur Prävention von Gesundheitsgefahren in der Lage, Warnungen auszusprechen oder Entscheidungen zu treffen, die ihren jeweiligen Aufgabenbereichen entsprachen. Bemerkenswert ist, dass ab Dezember 2019 die Meldungen der WHO zwar von der Presse, nicht aber von den Behörden an die Bevölkerung vermittelt worden sind. Die staatlichen Gesundheitsbehörden wie auch die Regierung, allen voran die damalige Gesundheitsministerin, kommunizierten im Gegenteil beruhigende Meldungen über die gute Vorbereitung und herausragende Qualität des nationalen Gesundheitswesens. Als Emmanuel Macron, am 16. März 2020, in seiner zweiten Ansprache im Rahmen des Krisenmanagement im martialischen Tonfall die Schließung von Schulen, Universitäten, kulturellen und nicht systemrelevanten Einrichtungen und gewissermaßen eine Quarantäne für die gesamten Bevölkerung ankündigte, blieben die Gesundheitsbehörden stumm. Die von Santé Publique France vorbereiteten Informationen über Abstands- und Hygieneregeln wurden durch Werbekampagnen in den Medien verbreitet, während die Mitteilungen französischer Regierungsbeamten über Masken und Tests unklar blieben.

Angesichts des Notstands richtete die zentrale Exekutive ad hoc Gremien der wissenschaftlichen Beratung und der Entscheidungsfindung ein und stellte diese an die Stelle der existierenden offiziellen Strukturen. Am 11. März 2020, einen Tag vor der offiziellen Anerkennung, dass Frankreich sich in einer Pandemie befindet, berief Emmanuel Macron einen wissenschaftlichen Rat, den Conseil scientifique Covid-19 und am 24. März 2020 ein Comité d'analyse, de recherche et d'expertise (CARE). Die Exekutive hat sich auf die beiden Beratungsinstanzen, jeweils mehrheitlich zusammengesetzt aus Medizinern und Wissenschaftler aus der biomedizinischen Forschung, in variierender Weise gestützt. In der ersten Phase der Krisenregulierung rechtfertigten die Regierung und der Staatschef die meisten ihrer Entscheidungen auf der Grundlage der Empfehlungen des Conseil scientifique Covid-19. ${ }^{18}$ Jedoch ab April 2020 und verstärkt in der zweiten und dritten Phase der Steuerungschronologie, hat die Exekutive wiederholt Abstand von dem Expertenwissen der beiden Beratungsgremien genommen. Sie beanspruchte die politische Verantwortung gegenüber den wissenschaftlichen Empfehlungen und hat daraufhin wiederholt gegen diese Empfehlungen der von ihr selbst einberufenen wissenschaftlichen Beratungsremien

\footnotetext{
${ }^{18}$ Vgl. Bergeron/Borraz/Castel/Dedieu 2020 (Fn. 8).
} 
entschieden. Hiermit führte der Präsident der Republik nicht nur vor Augen, dass er sich den Empfehlungen der Wissenschaftler zu widersetzen wisse und ausschließlich politische Entscheidungen zu treffen habe. Er ließ auch die Idee verbreiten, dass er selbst über epidemiologischen Sachverstand verfüge.

Die Aneignung der Kontrolle über alle Entscheidungen durch die zentrale Exekutive basiert im Wesentlichen auf der Mobilisierung eines verfassungsrechtlichen Instrument, des Conseil de Défense et de Sécurité nationale (CDSN), das der Staatspräsident zur Verteidigung der nationalen Sicherheit aktivieren kann. Auf dieser Basis richtete Emmanuel Macron den Conseil de Défense et de Sécurité sanitaire im März 2020 ein und schaffte dadurch eine Struktur derogativer Entscheidungsfindung mit direkter Anbindung an den Elysée-Palast. Als eine militärische Institution konzipiert stellt der CDSN einen eingeschränkten Ministerrat dar, den der Staatspräsident leitet und über dessen Besetzung er allein bestimmt. Der Conseil de Défense et de Sécurité sanitaire stellte den Ort aller wichtigen Entscheidungen im Krisenmanagement dar. ${ }^{19}$ Darüber hinaus wurden interministerielle Strukturen geschaffen, z. B. ein interministerieller Krisenstab, der zunächst im Gesundheitsministerium und dann im Innenministerium unter der Aufsicht des Premierministers angesiedelt war. Trotz der territorialen Differenzierungen, die am Ende der ersten Phase des Krisenmanagements einsetzte, und trotz des gelegentlichen Rückgriffs auf eine Kooperation mit den Bürgermeistern, lässt sich hier eine stätige Konzentration der Entscheidungsfindung bei der zentralen Exekutive beobachten.

\section{Die Instrumente des Krisenmanagements und ihre juristische Grundlage im} Fadenkreuz der Kritik

Die außerordentlichen Strukturen der Entscheidungsfindung und Kompetenzkonzentration haben ab März 2020 zu einer Reihe von Restriktionen geführt und rechtliche Formen angenommen, die einen so derogativen Charakter haben wie die Strukturen selbst.

Die Eindämmungsmaßnahmen und Kontaktbeschränkungen zum Schutz der Gesundheit der Bevölkerung in der ersten Phase des Krisenmanagements mündeten in einen Lockdown (17. März 2020 - 11. Mai 2020), der einen der striktesten in Europa darstellte. Neben der Schließung von Schulen und Universitäten, Restaurants, Kultureinrichtungen, der dringenden Empfehlung zum Homeoffice etc. wurde die Bewegungsfreiheit der Bevölkerung auf den

\footnotetext{
${ }^{19}$ Bandelow./Hassenteufel/Hornung 2021 (Fn. 4); Roussellier, Nicolas 2021: Le Conseil de Défense: dérive autoritaire ou régime de nécessité, in: AOC vom 15.01.2021. https://aoc.media/analyse/2021/01/14/le-conseilde-defense-derive-autoritaire-ou-regime-de-necessite/ (23.07.2021)
} 
Radius von tausend Metern zur eigenen Wohnung und auf eine Stunde am Tag eingeschränkt. Streng genommen handelte es sich bei diesen Regeln nicht um Maßnahmen des Gesundheitsschutzes, sondern um Maßnahmen der zivilen Sicherheit, die in den Zuständigkeitsbereich des französischen Innenministeriums fielen und deren Einhaltung von der Polizei kontrolliert wurde.

Die Restriktionen des ersten Lockdowns, im Französischen als confinement bezeichnet, der letztendlich die gesamte Bevölkerung in Quarantäne stellte, wie auch alle weiteren Eindämmungsmaßnahmen bis zum 1. Juni 2021 basierten auf der Ausrufung eines gesundheitlichen Notstands (état d'urgence sanitaire). Das entsprechende Gesetz, das so genannte Gesundheitsnotstandsgesetz, wurde am 23. März 2020 verabschiedet, wenige Tage nach dem Inkrafttreten des ersten Lockdowns. ${ }^{20}$ Das Notstandsgesetz hat der zentralen Exekutive ermöglicht, die Kontrolle über das gesamte Gesundheitssystem zu übernehmen und die Präfekten mit der Um- und Durchsetzung des Krisenmanagements auf dem gesamten Hoheitsgebiet zu beauftragen. Zudem erlaubte es der Regierung, den regulären Gesetzgebungsprozess bezüglich aller Dimensionen der Gesundheitskrise durch Exekutivdekrete zu ersetzen. Die Konzentration der Macht in den Händen der zentralen Exekutive galt zunächst für zwei Monate. Das Notstandsgesetz wurde jedoch mehrfach und schließlich bis zum 1. Juni 2021 verlängert. ${ }^{21}$. Seit dem 1. Juni 2021 gilt eine Übergangsregelung, die die Regierung ermächtigt, außergewöhnliche Maßnahmen zu ergreifen, wie etwa die Verlängerung des Gesundheitsnotstands im Département Guyana bis zum 30. September 2021.

In der Reaktion auf die Covid-19-Pandemie vervielfachten sich die Gesetze, Dekrete und Verordnungen für eine große Anzahl von Bereichen des öffentlichen Handelns: im Gesundheitswesen, in der Erstattung von Gesundheitskosten, bezüglich begleitender Maßnahmen für das Gesundheitspersonal, der Anpassung des Bildungssystems, der Organisation von Gottesdiensten und Beerdigungen, der Behandlung chronischer Krankheiten und psychischer Störungen, im Hinblick auf die Reorganisation des öffentlichen Verkehrs etc. Angesichts der Vielzahl von Regelungen zur Anpassung an und Reaktion auf die Pandemie haben zahlreiche institutionelle Akteure und private Organisationen der Öffentlichkeit ein juristisches Monitoring und regelmäßige Informationen sowie Erläuterungen zu den

\footnotetext{
${ }^{20}$ LOI n² 2020-290 du 23 mars 2020 d'urgence pour faire face à l'épidémie de COVID-19.

${ }^{21}$ LOI n 2021-160 du 15 février 2021 prorogeant l'état d'urgence sanitaire.
} 
verschiedenen Maßnahmen im Zusammenhang mit der Reaktionen auf Covid-19 im Internet zur Verfügung gestellt. ${ }^{22}$

Die Erläuterungstexte betreffen Maßnahmen im Gesundheitsschutz (z. B. Anweisungen zur Prävention von Infektionsrisiken), rechtliche Regelungen der Arbeit im Homeoffice und der öffentlichen Online-Dienste, die Möglichkeiten für Haus- und Familienpflege oder Pflegedienste wie auch die finanziellen Ausgleichsmaßnahmen für Berufsgruppen, die aufgrund der Pandemie ihrer Arbeit nicht nachgehen konnten. Darüber hinaus wurde über die Anpassungen im System der sozialen Sicherheit (Ausweitung der Ansprüche auf Arbeitslosenversicherung, Erstattung für Tests oder Pflege) informiert.

Das dichte und ineinander verschränkte Maßnahmenbündel hat zudem eine Vielzahl von politischen Reaktionen hervorgerufen, die hier nur resümierend skizziert werden können. Erstens weckten die Verschwommenheit und Widersprüchlichkeit der Regierung in Bezug auf die Nützlichkeit der Masken und Tests Zweifel daran, dass das französische Gesundheitssystem auf die Pandemie vorbereitet ist, ja an der Glaubwürdigkeit der staatlichen Erklärungen schlechthin. Das Vertrauen der Franzosen in ihre Regierung lag im April 2020 bei $32 \%$, hingegen in Großbritannien bei $61 \%$ und in Deutschland bei $60 \% .^{23}$ Das Misstrauen spiegelt sich in konkreter Form in den fast hundert beim Haute Cour de la Justice de la République (Gericht für Verfahren gegen den Staatspräsidenten und Regierungsmitglieder) wider. Mehrere hundert Klagen gingen zudem bei der Pariser Staatsanwaltschaft ein. Die parlamentarische Opposition bewirkte zwei Parlamentarische Untersuchungsausschüsse und schließlich beauftragte der Präsident der Republik selbst einen Genfer Virologen, Didier Pittet, mit einer Untersuchungskommission, die „das Management der Covid-19-Krise und die Antizipation von Pandemierisiken“ in dem sogenannten Pittet-Bericht evaluiert hat. ${ }^{24}$ Des Weiteren lässt sich in der Kritik ein spezieller Fokus auf die Einschränkung der individuellen Freiheiten im Zusammenhang mit dem Covid-19-Krisenmanagement ausmachen. In diesem Kontext sind zwei sich parallel entwickelnde Kritiklinien zu unterscheiden: zum einen eine, die aus populistischen Positionen hervorgeht und teilweise mit

\footnotetext{
22 Z. B. Textes réglementaires. https://travail-emploi.gouv.fr/le-ministere-en-action/coronavirus-covid19/article/textes-reglementaires (26.07.2021); Village de la justice. La communauté des métiers du droit: Veille légale et réglementaire - Crise sanitaire Covid-19, l'état du droit quotidien: https://www.villagejustice.com/articles/veille-legale-reglementaire-sur-crise-sanitaire-covid-etat-droit (26.07.2021).

${ }^{23}$ Cevipof 2020: Baromètre de la confiance politique - Vague 11bis, spéciale Coronavirus, Paris, S. 17. https://www.sciencespo.fr/cevipof/sites/sciencespo.fr.cevipof/files/OpinionWay\%201pour\%20le\%20CEVIPOFSowell-Barome\%cc\%80tre\%20de\%20la\%20confiance\%20en\%20politique\%20\%20vague11b\%20Comparaison\%20(1)-1-1.pdf (23.07.2021).

${ }^{24}$ Pittet, Didier (Hrsg.) 2021: Rapport final - Mission indépendante nationale sur l'évaluation de la gestion de la crise Covid-19 et sur l'anticipation des risques pandémiques. Rapport au Président de la République. https://www.vie-publique.fr/sites/default/files/rapport/pdf/279851.pdf (23.07.2021).
} 
verschwörungstheoretischen Argumenten arbeitet, zum anderen eine, die in der Tradition der Verteidigung individueller Freiheiten und Menschenrechte steht. Die populistische Kritik wird vor allem von der linken Partei La France Insoumise getragen, deren Parteiführer JeanLuc Mélenchon ist und die 17 von insgesamt 577 Abgeordneten in der Nationalversammlung stellt. La France Insoumise stellt die einzige im Parlament vertretene Partei dar, die frontal gegen die Regierung opponiert. Sie fokussiert in ihrer Opposition auf den Autoritarismus des Zentralstaates und bemüht hierbei mitunter eine verschwörungstheoretische Argumentation. Marine Le Pens Rassemblement National liebäugelte zu Beginn der Pandemiekrise mit ähnlichen Positionen, die die Partei jedoch aufgrund ihres Strebens nach parlamentarischer Respektabilität wiederaufgegeben hat. Der Widerstand gegen den Gesundheitspass, den die Regierung ab August 2021 für den Zugang zu Gastronomie, Kulturveranstaltungen und Fernverkehr einführen wird, hat in der gegenwärtigen dritten Phase der Steuerungschronologie zu einer heterogenen Koalition aus Anhängern von Jean-Luc Mélenchons France Insoumise, von rechtsextremen (ansonsten für das Parteienspektrum unbedeutenden) Parteiführern und von Anhängern der wiederauflebenden Bewegung der Gilets jaunes („Gelbwesten“) geführt. Die Bewegung letzterer hat sich im Herbst und Winter 2018-2019 herausgebildet, um gegen die zentralistische Regierungspolitik und die territorialen Ungleichheiten zu protestieren. ${ }^{25}$

Was die zweite Kritiklinie anbelangt, so haben sich ein Teil der nationalen Presse (z. B. Le Monde, Médiapart, Libération) wie auch die staatliche Instanzen bzw. Gremien und Organisationen, die für die bürgerlichen Freiheiten eintreten, für die Verteidigung der individuellen Freiheiten und der Bekämpfung von Diskriminierungen eingesetzt. Der Défenseur des Droits, eine Obudsinstanz, die in Frankreich für die Beilegung von Streitigkeiten zwischen Bürgern und dem Staatsapparat zuständig ist, wie auch in der Öffentlichkeit anerkannte Organisationen, etwa die Ligue des Droits de l'Homme, haben immer wieder ihre Erschütterung über das Ausmaß und die Dauer der Ausnahmegesetze wie auch ihre Kritik an der Aussetzung der individuellen Rechte und Freiheiten durch diese Gesetze zum Ausdruck gebracht. ${ }^{26}$ Den Vertretern dieser zweiten Kritiklinie ist es sicherlich weniger darum gegangen, eine politische Opposition zur Regierungspolitik herauszubilden. Doch zielten sie darauf, die öffentliche Debatte zu beeinflussen und eine andere Gewichtung

\footnotetext{
${ }^{25}$ vgl. Halmes, 2020 (Fn. 11).

${ }^{26}$ Défenseur des droits: Covid-19 et urgences sanitaire, le rôle du défenseur des droits. Protéger les droits et libertés durant la crise. https://www.defenseurdesdroits.fr/fr/covid-19-et-urgence-sanitaire-le-role-dudefenseur-des-droits (26.07.2021); Ligue des droits de l'Homme: Etat d'urgence Covid-19 https://www.Idhfrance.org/tag/covid-19/ (24.07.2021)
} 
der Restriktionen wie auch die Einhaltung demokratischer und rechtsstaatlicher Verfahren zu erreichen. Zugleich haben sie immer wieder auf die sozialen Ungleichheiten sowohl in Bezug auf eine mögliche Infektion mit dem Sars-Cov-2-Virus als auch im Hinblick auf die Auswirkungen der Anti-Covid-Maßnahmen hingewiesen. ${ }^{27}$

Drittens sind das Krisenmanagement und die hierfür gewählten Instrumente des öffentlichen Handelns kritisiert worden. Wie oben erwähnt, hat die Regierung Ad-hoc-Organisationen zur Entscheidungsunterstützung eingerichtet statt auf die existierenden behördlichen Instanzen zurückzugreifen. Doch hat die hierdurch entstandene organisatorische Dichte, die zu der Komplexität der standardisierten Verwaltungsabläufe hinzukam, den Prozess der Entscheidungsfindung verlangsamt. Der oben erwähnte Pittet-Bericht weist deutlich auf die Probleme hin, die durch die Duplizierung einer Vielzahl von Entscheidungsstrukturen innerhalb des Gesundheitsapparats entstanden sind: „Die Organisation des Krisenmanagements innerhalb des Gesundheitsministeriums hat sich wiederholt so entwickelt, dass spezielle Einheiten (Versorgung und Tests) ,neben` dem Krisenzentrum für Gesundheit [...] entstanden.“28. So wurden zum Beispiel aufgrund von dysfunktionalen Abläufen im Verwaltungsapparat die Kapazitäten der dem medizinisch-biologischen System angegliederten Einrichtungen (Veterinärzentren, private Einrichtungen etc.) nicht für die Durchführung von Covid-Tests mobilisiert und die für die Durchführung einer großen Anzahl von Test nötigen Maschinen vom Gesundheitsministerium nicht vor Ende April 2020 bestellt. Ausreichende Testkapazitäten konnten in Frankreich erst ab Mitte Mai 2020 eingesetzt werden. Ebenfalls wird in dem Pittet-Bericht die Entscheidung, der Caisse d'Assurance Maladie, einer auf finanzielle Fragen spezialisierten Versicherungseinrichtung, die Nachverfolgung von infizierten Personen zu übergeben, als ineffizient bezeichnet.

\section{Konzentration und Multiplikation der Zuständigen, Personalisierung und Politisierung der Entscheidungsmacht}

Die soweit skizzierten kritischen Reaktionen verweisen auf einen Prozess, den das zentralistische und zugleich autoritative Krisenmanagement der französischen Exekutive vorangetrieben hat und der an die institutionalisierten Inkongruenzen in der territorialen Ordnung des französischen Staatsregimes anknüpft. Gekennzeichnet ist dieser Prozess durch

\footnotetext{
${ }^{27}$ Duvoux, Nicolas/Lelièvre, Michèle (Hrsg.) 2021: La pauvreté démultipliée. Dimensions, processus et réponses. Printemps 2020 | printemps 2021. Rapport au Premier Ministre du Conseil national des politiques de lutte contre la pauvreté et l'exclusion sociale. Paris. https://www.cnle.gouv.fr/IMG/pdf/rapport lutteexclusion fin.pdf (28.05.2021).

${ }^{28}$ Pittet 2021, S. 11 (Zitat übersetzt von den Autoren) (Fn. 26).
} 
eine Konzentration und Multiplikation der Zuständigkeiten wie auch durch eine Personalisierung und Politisierung der Steuerung des öffentlichen Handelns. Wie wir im ersten Teil unseres Beitrags gezeigt haben, führen die Dezentralisierungshürden, die in das territoriale Regime des französischen Staatssystems im öffentlichen Handeln und insbesondere im öffentlichen Gesundheitswesen zu vielfachen Überschneidungen von Zuständigkeiten, zu weitreichenden Redundanzen und vor allem zur Unfähigkeit der Behörden der Gebietskörperschaften, die Maßnahmen, für die sie verantwortlich sind, selbst zu finanzieren. Zudem produziert die Kompetenzverflechtung, neben einem erheblichen Verlust an Verwaltungseffizienz, für die Bürger intransparente Strukturen. In dieser Hinsicht hat die Covid-19-Pandemie die gesamte Inkohärenz in der Organisation des Verhältnisses zwischen Zentralstaat und Gebietskörperschaften wie auch in der Koordination des französischen Gesundheitswesens wie in einem Vergrößerungsglas vor Augen geführt, was ein parlamentarischer Untersuchungsbericht schon im Mai 2020 unterstrichen hat. ${ }^{29}$

Bemerkenswert ist an dieser Stelle, dass die französische Exekutive in ihren Antworten auf die Pandemie, wie die britische und US-amerikanische Regierung, von Beginn der Krise und verstärkt vom Schuljahr 2020/2021 an auf die Expertise privater Beratungsunternehmen zurückgegriffen hat. Der Presse, die über die parlamentarischen Debatten hinsichtlich des Kaufs dieser Expertise berichtet hat, ist zu entnehmen, dass in dem Zeitraum von März 2020 bis Februar 2021 sieben Beratungsfirmen beauftragt worden sind - und zwar für eine Summe von fast 12 Millionen Euro. Dieser Aspekt des Krisenmanagements, der mit der Machtkonzentration in den Ministerkabinetten verbunden ist, führt nicht zuletzt vor Augen, wie in dem antibürokratischen Streben der Staatsspitze nach Effizienz, Gremien der Expertise und Kontrolle im öffentlichen Handeln vervielfältigt und dysfunktionale Redundanzen geschaffen werden.

Die Personalisierung der Entscheidungsmacht durch die doppelköpfige Exekutive - den Präsidenten und Premierminister - während der Covid-19-Krise ist eine Folge der starken Zentralisierung des französischen Systems. So ist der Gesundheitsminister im Verlauf des Krisenmanagements zum Vollstrecker der Entscheidungen des Staatspräsidenten und seines Premierministers geworden ist. Gleichzeitig wurden die Gesundheitsbehörden und die wissenschaftlichen Beratungsinstanzen in der zweiten und dritten Phase der französischen Steuerungschronologie regelrecht ausgegrenzt. In Anbetracht der Unzulänglichkeit der Gesundheitsprävention und der Steuerung der Gesundheitsbehörden scheint die französische

\footnotetext{
${ }^{29}$ Sénat 2020: Rapport d'information sur les collectivités territoriales face au Covid-19. Rapport d'information $\mathrm{n}^{\circ}$ 625. http://www.senat.fr/rap/r19-625/r19-625.html (24.07.2021).
} 
Exekutive die personalisierende Zentralisierung der Entscheidungsmacht privilegiert zu haben. Möglicherweise glaubte sie, dadurch an Effizienz im Management der Covid-19-Krise zu gewinnen $^{30}$ und zugleich politischen Profit aus dem Krisenmanagement ziehen zu können. Doch haben der Staatspräsident und der Premierminister hierdurch die Tendenz zur Politisierung der Steuerungsprobleme in der Covid-19-Pandemie verstärkt, was nicht zuletzt die kritischen Reaktionen auf das Krisenmanagement andeuten.

Insgesamt gesehen machen die Konzentration und Multiplikation der Zuständigkeiten wie auch die Personalisierung und Politisierung der Steuerung des öffentlichen Handelns eine Entkopplung deutlich - und zwar zwischen den Orten, an denen Entscheidungen getroffen werden, und denen, an denen Gesundheitsprobleme oder andere Probleme hinsichtlich gesellschaftlicher Solidarität behandelt werden. Diese Entkoppelung verschärft die Spannungen zwischen den unterschiedlichen Akteuren des öffentlichen Handelns. In dem Krisenmanagement der Covid-19-Pandemie hat sie die Steuerungskapazitäten der beteiligten Akteure maßgeblich eingeschränkt und insofern den Vollzug relevanter Eindämmungsmaßnahmen behindert.

Die fehlende territoriale Differenzierung des Krisenmanagements ermöglichte zwar eine formale Gleichbehandlung der Bevölkerung, führte aber $\mathrm{zu}$ Missverständnissen und Spannungen mit bestimmten Vertretern der Gebietskörperschaften und trug letztendlich zu einer Schärfung sozialer Ungleichheiten bei. Zudem erlaubte die Schwäche der Steuerungskapazitäten der lokalen Behörden keine Kompensation der Widersprüche in der zentralistischen Pandemiebekämpfung, was in föderalen Staaten, sogar in den von Donald Trump regierten USA oder im von Jaïr Bolsonaro Brasilien, möglich war. Auf der horizontalen Ebene konnten die Spannungen zwischen der zentralen Entscheidungsebene und den öffentlichen wie privaten Akteuren der verschiedenen Sektoren des öffentlichen Handelns genauso wenig ausgeglichen werden. Sie sind in gleicher Weise durch das asymmetrische Verhältnis zwischen Zentrum-Peripherie behindert worden.

\footnotetext{
${ }^{30}$ Vgl. Bergeron/Borraz/Castel/Dedieu 2020 (Fn. 8).
} 